\title{
Оптические сечения поглощения и силы осцилляторов двойного донора магния в кремнии
}

\author{
( Ю.А. Астров ${ }^{1}$, Л.М. Порцель ${ }^{1}$, В.Б. Шуман ${ }^{1}$, А.Н. Лодыгин ${ }^{1}$, Н.В. Абросимов ${ }^{2}$, \\ С.Г. Павлов ${ }^{3}$, H.-W. Hübers ${ }^{3,4}$ \\ ${ }^{1}$ Физико-технический институт им. А.Ф. Иоффе Российской академии наук, \\ 194021 Санкт-Петербург, Россия \\ ${ }^{2}$ Leibniz-Institut für Kristallzüchtung (IKZ), \\ Berlin, Germany \\ ${ }^{3}$ Institute of Optical Sensor Systems, German Aerospace Center (DLR), \\ Berlin, Germany \\ ${ }^{4}$ Institut für Physik, Humboldt-Universität zu Berlin, \\ Berlin, Germany \\ E-mail: yuri.astrov@mail.ioffe.ru
}

Поступила в Редакцию 1 декабря 2020 г.

В окончательной редакции 11 декабря 2020 г.

Принята к публикации 11 декабря 2020 г.

\begin{abstract}
Изучены оптические свойства примеси магния в кремнии, атомы которого в межузельных положениях в решетке являются глубокими двойными донорами с энергией ионизации 107.56 мэВ в нейтральном состоянии. Для оптических переходов из основного состояния нейтрального центра на возбужденные уровни $2 p_{0}$ и $2 p_{ \pm}$определены сечения поглощения и силы осциллятора. Эти параметры рассчитаны из спектров примесного поглощения в образцах с различной концентрацией магния, измеренных при температуре $T \approx 5 \mathrm{~K}$. Содержание глубокого донора в образцах определялось с помощью измерений эффекта Холла в диапазоне температуры $78-300 \mathrm{~K}$. Полученные характеристики внутрицентровых переходов магния сравниваются с соответствующими литературными данными для мелких доноров V группы в кремнии, которые являются примесями замещения. Обнаружено, что оптические характеристики исследованных переходов в магнии согласуются с зависимостями соответствующих величин от энергии ионизации мелких доноров, экстраполированными в область бо́льших энергий связи электрона.
\end{abstract}

Ключевые слова: кремний, глубокие доноры, магний, оптическая спектроскопия.

DOI: 10.21883/FTP.2021.04.50728.9564

\section{1. Введение}

Определение содержания электрически активных примесей в полупроводниках путем измерения спектров поглощения света является удобным диагностическим методом. Получение количественных результатов этим способом возможно, если известна связь между концентрацией примеси и параметрами спектра поглощения материала в области энергий фотонов, взаимодействующих с центрами примеси. Соответствующие калибровки выполнены, в частности, для ряда однозарядных, близких к водородоподобным доноров в кремнии, которые создаются примесями замещения [1-4]. В этих исследованиях определены величины сечений поглощения для некоторых дипольно-разрешенных внутрицентровых переходов из основного состояния в нечетные возбужденные. При этом установлено, что с увеличением энергии ионизации донора в ряду $\mathrm{Sb}, \mathrm{P}, \mathrm{As}, \mathrm{Bi}$ сечение поглощения в то же самое возбужденное состояние монотонно уменьшается. Такая закономерность находит естественную интерпретацию: степень локализации волновой функции основного состояния центра увеличивается с ростом энергии ионизации, что приводит к снижению пространственного перекрытия волновых функций основного и возбужденного состояний и соответственно к уменьшению матричных элементов взаимодействия электромагнитного излучения с центром.

Данные о сечениях поглощения для глубоких многоэлектронных центров в кремнии менее полные. Вместе с тем получение детальной информации об их оптических свойствах важно, в частности, в связи с интересом к развитию устройств кремниевой фотоники, где могут быть использованы свойства глубоких центров $[5,6]$. Межузельный магний $\mathrm{Mg}_{i}$ является специфическим донором с набором свойств, типичных для двойных доноров, образованных узельными примесями, а также сильно отличающихся от них. Так, его энергия ионизации, $E_{\mathrm{Mg}}=107.56$ мэВ, как это определено из спектров примесного поглощения [7], является промежуточной между энергиями ионизации одноэлектронных доноров (42-71 мэВ), которые формируются примесями замещения V группы, и двухэлектронных доноров VI группы (199-319 мэВ), также занимающих узельные положения в решетке. С другой стороны, такой центр обнаруживает типичные свойства двойного гелиеподобного донора [6-8], формируя разные зарядовые состояния. В связи с таким особым положением межузельного донора $\mathrm{Mg}_{i}$ представляет интерес получение количественных данных о его сечениях поглощения и силах осцилляторов для оптических переходов. В настоящем 
сообщении представлены соответствующие данные для некоторых внутрицентровых переходов центра $\mathrm{Mg}_{i}$ в кремнии. Они сравниваются с известными данными для узельных одноэлектронных доноров в кремнии.

\section{2. Полученные результаты}

В опытах использовались образцы $\mathrm{Si}: \mathrm{Mg}$, полученные диффузией примеси с помощью сандвич-метода, как это описано в [9-11]. В качестве исходного материала служил монокристаллический кремний с малым содержанием остаточных мелких доноров и акцепторов, выращенный методом зонной плавки (см. табл. 1). Была изготовлена серия образцов с разным содержанием $\mathrm{Mg}_{i}$. Концентрация межузельного магния определялась из измерений эффекта Холла в температурной области $T=78-300 \mathrm{~K}$ с последующей обработкой данных в модели, учитывающей статистику двойного донора (см. $[12,13]$ и ссылки в этих работах).

Спектры поглощения образцов $\mathrm{Si}: \mathrm{Mg}$ толщиной 1-2 мм измерялись с использованием фурье-спектрометра, модель Bruker Vertex 80v, при типичном спектральном разрешении $0.25 \mathrm{~cm}^{-1}$. Источником инфракрасного излучения в установке служил глобар. В зависимости от исследуемого спектрального диапазона применялись различные приемники излучения. Калибровка спектров поглощения $\mathrm{Mg}_{i}$ осуществлялась с помощью нормировки, учитывающей поглощение кремния в области двухфононных решеточных процессов, спектрально не пересекающейся с областями внутрицентровых переходов как одноэлектронных, так и двухэлектронных доноров.

Экспериментальную величину поглощения излучения в образце в пределах линии возбуждения центра можно охарактеризовать интегральным коэффициентом поглощения перехода $(A)$ :

$$
A=\int_{\text {line }} \alpha(v) d v,
$$

где $\alpha(v)$ - измеряемый коэффициент поглощения (в $\left.\mathrm{cm}^{-1}\right), v-$ волновое число излучения $\left(\right.$ в $\left.\mathrm{cm}^{-1}\right)$, интегрирование проводится в пределах спектральной области линии поглощения.

Из величины $A$ и концентрации центров $N\left(\mathrm{~cm}^{-3}\right)$ можно определить интегральное сечение поглощения перехода $(\Sigma)$ :

$$
\Sigma=\frac{A}{N}
$$

Зная величину $\Sigma$ внутрицентрового перехода, можно из оптических экспериментов определить концентрацию примеси. Очевидно, что для правильного определения параметра $\Sigma$ образец должен быть оптически тонким в спектральной области поглощения света, т.е. поглощение излучения зондирующего пучка света спектрометра должно зависеть линейно от концентрации исследуемых примесных центров.

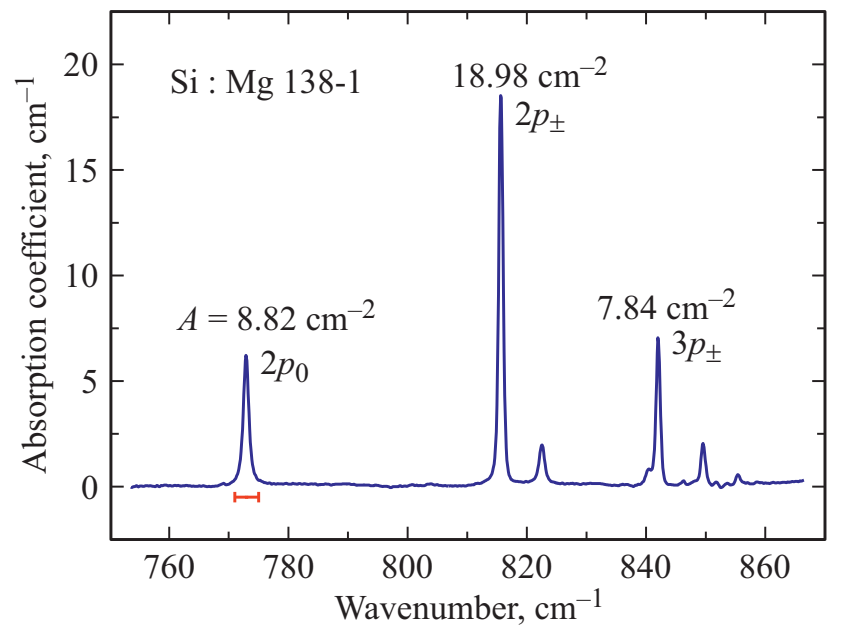

Рис. 1. Спектр поглощения образца 138-1 при температуре $\sim 5 \mathrm{~K}$ в области переходов из основного в возбужденные состояния межузельного магния. Для наиболее интенсивных линий отмечены полученные значения интегральных коэффициентов поглощения. Область интегрирования сигнала для всех линий составила $4 \mathrm{~cm}^{-1}$ (отмечено для перехода в состояние $\left.2 p_{0}\right)$.

На рис. 1 показан типичный спектр поглощения образца $\mathrm{Si}: \mathrm{Mg}$, измеренный при температуре $\sim 5 \mathrm{~K}$. Как видно, наиболее сильные линии поглощения соответствуют переходам из основного $1 s\left(A_{1}\right)$ в возбужденные состояния $2 p_{0}, 2 p_{ \pm}$и $3 p_{ \pm}$. На рисунке отмечены значения интегральных коэффициентов поглощения для этих линий, определенные с использованием соотношения (1). При определении значений $A$ линий спектра область интегрирования составляла $\Delta v=4 \mathrm{~cm}^{-1}$ (показано на рисунке для линии $2 p_{0}$ ). Отметим, что измеренная ширина указанных спектральных линий $\mathrm{Si}: \mathrm{Mg}$ заметно превышает значения, наблюдаемые для внутрицентровых переходов мелких доноров, а также глубоких доноров халькогенов в Si. Данное обстоятельство отмечалось в работе [14]. Возможно, что уширение линий поглощения в $\mathrm{Si}: \mathrm{Mg}$ вызывается механическими напряжениями в решетке полупроводника, которые обусловлены значительной концентрацией магния в электрически неактивном состоянии (см. [15]).

В табл. 1 приведены характеристики поглощения инфракрасного излучения для двух линий $\mathrm{Mg}_{i}^{0}$, определенные для четырех образцов, параметры исходного кремния (тип легирования, удельное сопротивление $\rho$ ) и концентрация межузельного магния $\left(N_{\mathrm{Mg}}\right)$.

На рис. 2 показаны величины интегральных сечений поглощения для перехода в состояние $2 p_{0}$, полученные для образцов с разной концентрацией $\mathrm{Mg}$. Наблюдается разброс экспериментальных значений, что связано с погрешностями определения как абсолютных значений поглощения излучения, так и концентрации примеси. Вместе с тем видно, что данные группируются вокруг среднего значения $\sim 7.7 \cdot 10^{-15}$ см. Переходу в состояние $2 p_{ \pm}$соответствует усредненное значение 
Таблица 1. Исследованные образцы и полученные данные

\begin{tabular}{c|c|c|c|c|c|c}
\hline $\begin{array}{c}\text { Номер } \\
\text { образца }\end{array}$ & $\begin{array}{c}\text { Легирование } \mathrm{Si}, \\
\rho, \mathrm{Oм} \cdot \mathrm{cm}\end{array}$ & $\begin{array}{c}N_{\mathrm{Mg}}, \\
\mathrm{cm}^{-3}\end{array}$ & $\begin{array}{c}A_{2 p_{0}}, \\
\mathrm{~cm}^{-2}\end{array}$ & $\begin{array}{c}A_{2 p_{ \pm}}, \\
\mathrm{cm}^{-2}\end{array}$ & $\begin{array}{c}\Sigma_{2 p_{0}}, \\
\mathrm{~cm}\end{array}$ & $\begin{array}{c}\Sigma_{2 p_{ \pm}}, \\
\mathrm{cm}\end{array}$ \\
\hline $138-1$ & $n-\mathrm{Si}, 8 \cdot 10^{3}$ & $1.15 \cdot 10^{15}$ & 8.82 & 18.98 & $7.7 \cdot 10^{-15}$ & $1.7 \cdot 10^{-14}$ \\
$90-2$ & $p-\mathrm{Si}, 3.1 \cdot 10^{3}$ & $9.5 \cdot 10^{14}$ & 6.94 & 12.56 & $7.3 \cdot 10^{-15}$ & $1.3 \cdot 10^{-14}$ \\
$85-3$ & $p-\mathrm{Si}, 2 \cdot 10^{3}$ & $8.2 \cdot 10^{14}$ & 7.38 & 20.58 & $8.9 \cdot 10^{-15}$ & $2.5 \cdot 10^{-14}$ \\
$81-3$ & $p-\mathrm{Si}, 3.1 \cdot 10^{3}$ & $4.7 \cdot 10^{14}$ & 3.3 & 9.19 & $7.0 \cdot 10^{-15}$ & $2.0 \cdot 10^{-14}$ \\
\hline
\end{tabular}

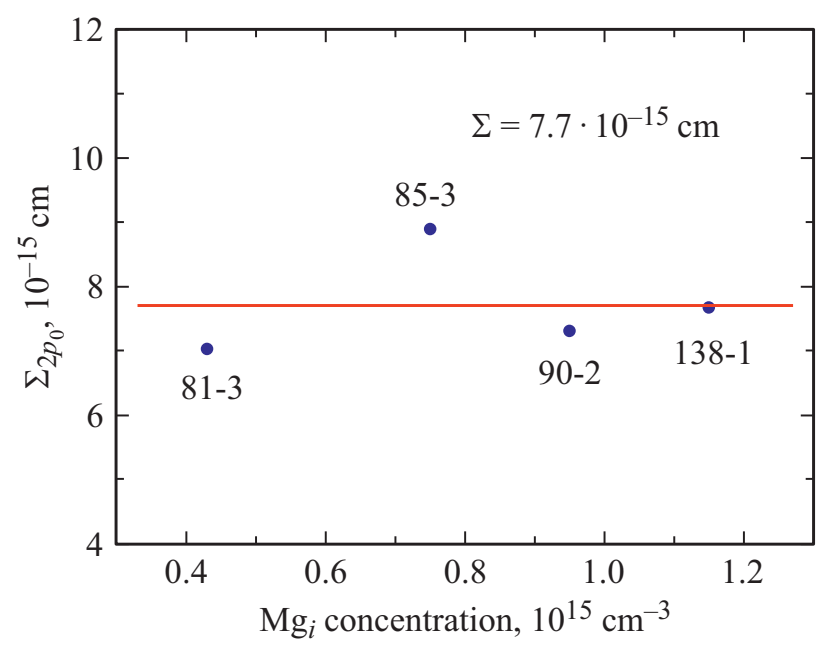

Рис. 2. Величины интегральных сечений поглощения для перехода из основного состояния межузельного магния на уровень $2 p_{0}$, полученные для серии образцов с различной концентрацией примеси (табл. 1). Среднее значение сечения показано горизонтальной линией.

$\sim 2 \cdot 10^{-14}$ см. Однако в связи с большим значением сечения поглощения условие оптически тонкого образца начинает нарушаться для переходов в состояние $2 p_{ \pm}$; данная экспериментальная проблема известна также при изучении мелких примесей в кремнии.

Важной характеристикой примесного центра, определяющей взаимодействие с ним электромагнитного излучения, является сила осциллятора перехода $f$, а также производная от этого параметра - дипольный матричный элемент перехода. Величина $f$ представляет собой отношение интенсивности переходов между энергетическими состояниями данной квантовой системы под воздействием излучения и интенсивности перехода так называемого классического одноэлектронного атома $[16,17]$. Силу осциллятора спектральной линии можно определить из интегральных сечений поглощения перехода. Для доноров в кремнии ее значение можно получить из соотношения $[2,4,18]$

$$
f=\frac{n c \bar{m}}{2 \pi^{2} \hbar e^{2}} \frac{1}{N} \int_{\text {line }} \alpha(v) d v=\frac{9.88 \cdot 10^{11}}{N} \int_{\text {line }} \alpha(v) d v
$$

где $n$ - показатель преломления кремния в данной спектральной области, $n \approx 3.38 \cdot\left(1+3.9 \cdot 10^{-5} \cdot T\right)$, $\bar{m}=\left[\left(2 m_{t}^{-1}+m_{l}^{-1}\right) / 3\right]^{-1}-$ усредненная эффективная масса электрона, $m_{t}$ и $m_{l}-$ поперечная и продольная массы в долине зоны проводимости, $c, \hbar$ и $e-$ скорость света, постоянная Планка и заряд электрона соответственно. Размерность численного коэффициента перед интегралом $\left(\mathrm{cm}^{-1}\right)$.

Теоретические расчеты сил осцилляторов $f$ переходов из основного состояния на уровни $2 p_{ \pm}$и $2 p_{0}$ для мелких доноров V группы в кремнии приведены в статьях $[2,19]$. Расчеты выполнены в приближении эффективной массы (EMA). На рис. 3 точками 1 показаны значения $f$, полученные в этих работах. Данные представлены в двойном логарифмическом масштабе как зависимость величины $f$ от энергии ионизации примеси. Сплошные линии соответствуют аппроксимациям этих зависимостей степенными функциями. С увеличением энергии ионизации примеси наблюдается уменьшение значения $f$. Этот факт авторы [19] объясняют увеличением степени локализации состояний центра.

Рис. 3 содержит силы осцилляторов, рассчитанные по формуле (3) с использованием экспериментальных значений $\Sigma$ для мелких доноров $\mathrm{Sb}, \mathrm{P}$, As в кремнии. Величины $\Sigma$ и ссылки на оригинальные публикации, из которых взяты эти данные, приведены в табл. 2, где также содержатся расчетные значения $f$.

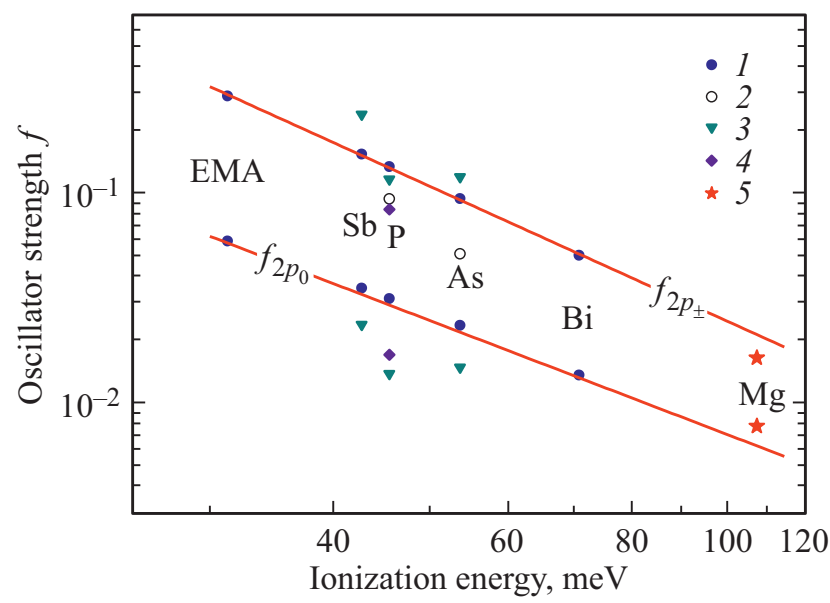

Рис. 3. Зависимость силы осцилляторов переходов $2 p_{ \pm}$и $2 p_{0}$ от энергии ионизации для ряда доноров в кремнии. $1-$ работы [2,19], $2-[1], 3-[3], 4-[4], 5-$ настоящая работа, образец 138-1. Сплошные линии - аппроксимации зависимостей $f$ от энергии ионизации $[2,19]$ с помощью степенных функций. 
Таблица 2. Значения интегральных сечений поглощения $\Sigma$ и сил осцилляторов переходов $f$ для мелких доноров $\mathrm{V}$ группы в кремнии, рассчитанные по данным ряда работ с использованием формулы (3), а также полученные в настоящей работе $\Sigma$ и $f$ для $\mathrm{Mg}_{i}$ (образец 138-1)

\begin{tabular}{|c|c|c|c|c|c|c|c|c|}
\hline \multirow{2}{*}{$\begin{array}{l}\text { Переходы, } \\
\text { источники }\end{array}$} & \multicolumn{2}{|c|}{$\mathrm{Sb}$} & \multicolumn{2}{|c|}{$\mathrm{P}$} & \multicolumn{2}{|c|}{ As } & \multicolumn{2}{|c|}{ Si:Mg 138-1 } \\
\hline & $\Sigma, \mathrm{cm}$ & $f$ & $\Sigma, \mathrm{cm}$ & $f$ & $\Sigma, \mathrm{cm}$ & $f$ & $\Sigma, \mathrm{cm}$ & $f$ \\
\hline $\begin{array}{l}2 p_{ \pm}[1] \\
2 p_{0}[3] \\
2 p_{ \pm}[3] \\
2 p_{0}[4] \\
2 p_{ \pm}[4] \\
2 p_{0} \\
2 p_{ \pm}\end{array}$ & $\begin{array}{l}2.4 \cdot 10^{-14} \\
2.4 \cdot 10^{-13}\end{array}$ & $\begin{array}{l}0.0235 \\
0.235\end{array}$ & $\begin{array}{r}9.4 \cdot 10^{-14} \\
1.4 \cdot 10^{-14} \\
1.18 \cdot 10^{-13}\end{array}$ & $\begin{array}{l}0.093 \\
0.014 \\
0.116 \\
0.0169 \\
0.0829\end{array}$ & $\begin{array}{r}5.2 \cdot 10^{-14} \\
1.5 \cdot 10^{-14} \\
1.21 \cdot 10^{-13}\end{array}$ & $\begin{array}{l}0.051 \\
0.015 \\
0.119\end{array}$ & $\begin{array}{l}7.7 \cdot 10^{-15} \\
1.6 \cdot 10^{-14}\end{array}$ & $\begin{array}{l}0.0076 \\
0.0163\end{array}$ \\
\hline
\end{tabular}

Там же представлены значения $f$, полученные в настоящей работе для образца 138-1. Силы осцилляторов определялись по формуле (3) и значениям $\Sigma$ из табл. 1. Величины $f$ исследованных спектральных линий магния хорошо ложатся на теоретическую зависимость $f(E)$ для мелких доноров в кремнии, экстраполированную в сторону бо́льших значений энергии ионизации. Заметим, что для линии $2 p_{0}$, где абсолютное значение поглощения света ниже по сравнению с соответствующим значением для уровня $2 p_{ \pm}$и соответственно снижена роль нелинейных эффектов при измерениях, точность определения сечения поглощения и силы осциллятора выше.

\section{3. Заключение}

Таким образом, в настоящем исследовании определены сечения поглощения для наиболее интенсивных внутрицентровых переходов межузельных атомов магния в кремнии, которые находятся в нейтральном состоянии. Результаты могут использоваться для бесконтактного определения концентрации электрически активного магния в образцах $\mathrm{Si}: \mathrm{Mg}$ с помощью оптической спектроскопии. С использованием экспериментальных данных рассчитаны также силы осцилляторов $f$ оптических переходов в состояния $2 p_{ \pm}$и $2 p_{0}$ магния. Результаты сопоставлены с соответствующими данными для доноров V группы в кремнии, которые получены путем обработки опубликованных в литературе спектров поглощения этих мелких доноров. Найдено, что значения $f$ для глубокого донора магния в нейтральном состоянии соответствуют тенденции изменения этой величины, которая наблюдается при увеличении энергии ионизации мелких однозарядных доноров V группы в кремнии.

\section{Финансирование работы}

Исследование поддержано программой ФТИ им. А.Ф. Иоффе РАН, № 0040-2019-0016.

\section{Конфликт интересов}

Авторы заявляют, что у них нет конфликта интересов.

\section{Список литературы}

[1] S.Ch. Baber. Thin Sol. Films, 72, 201 (1980).

[2] L. Beĭnikhes, Sh.M. Kogan. Sov. Phys. JETP, 66 (I), 164 (1987) [Zh. Eksp. Teor. Fiz., 93, 285 (1987)].

[3] E. Rotsaert, P. Clauws, J. Vennik, L. Van Goethem. J. Appl. Phys., 65, 730 (1989).

[4] B.A. Andreev. Mater. Sci. Forum, 196-201, 121 (1995).

[5] A. De Abreu, C. Bowness, R.J.S. Abraham, A. Medvedova, K.J. Morse, H. Riemann, N.V. Abrosimov, P. Becker, H.-J. Pohl, M.L.W. Thewalt, S. Simmons. Phys. Rev. Appl., 11, 044036 (2019).

[6] В.Н. Шастин, Р.Х. Жукавин, К.А. Ковалевский, В.В. Цыпленков, В.В. Румянцев, Д.В. Шенгуров, С.Г. Павлов, В.Б. Шуман, Л.М. Порцель, А.Н. Лодыгин, Ю.А. Астров, Н.В. Абросимов, J.M. Klopf, H.-W. Hübers. ФТП, 53, 1263 (2019).

[7] L.T. Ho, A.K. Ramdas. Phys. Rev. B, 5, 462 (1972).

[8] A.-L. Thilderkvist, M. Kleverman, H.-G. Grimmeiss. Phys. Rev. B, 49, 16338 (1994).

[9] R.K. Franks, J.B. Robertson. Solid State Commun., 5, 479 (1967).

[10] В.Б. Шуман, Ю.А. Астров, А.Н. Лодыгин, Л.М. Порцель. ФТП, 51, 1075 (2017).

[11] Yu.A. Astrov, V.B. Shuman, L.M. Portsel, A.N. Lodygin, S.G. Pavlov, N.V. Abrosimov, V.N. Shastin, H.-W. Hübers. Phys. Status Solidi A, 214, 1700192 (2017).

[12] Yu.A. Astrov, L.M. Portsel, A.N. Lodygin, V.B. Shuman. Semicond. Sci. Technol., 26, 055021 (2011).

[13] S.G. Pavlov, N. Deßmann, A. Pohl, V.B. Shuman, L.M. Portsel, A.N. Lodygin, Yu.A. Astrov, S. Winnerl, H. Schneider, N. Stavrias, A.F.G. van der Meer, V.V. Tsyplenkov, K.A. Kovalevsky, R.Kh. Zhukavin, V.N. Shastin, N.V. Abrosimov, H.W. Hübers. Phys. Rev. B, 94, 075208 (2016).

[14] R.J.S. Abraham, A. De Abreu, K.J. Morse, V.B. Shuman, L.M. Portsel, A.N. Lodygin, Yu.A. Astrov, N.V. Abrosimov, S.G. Pavlov, H.-W. Hübers, S. Simmons, M.L.W. Thewalt. Phys. Rev. B, 98, 02202 (2018).

[15] Л.М. Порцель, В.Б. Шуман, А.А. Лаврентьев, А.Н. Лодыгин, Н.В. Абросимов, Ю.А. Астров. ФТП, 54, 331 (2020).

[16] Н.А. Ярыкин, В.Б. Шуман, Л.М. Порцель, А.Н. Лодыгин, Ю.А. Астров, Н.В. Абросимов, J. Weber. ФТП, 53, 799 (2019).

[17] R.C. Hilborn. Am. J. Phys., 50, 982 (1982). 
[18] D.L. Dexter. Solid State Phys., 6, 355 (1958).

[19] P. Clauws, J. Broeckx, E. Rotsaert, J. Vennik. Phys. Rev. B, 38, 12377 (1988).

Редактор Л.В. Шаронова

\section{Optical cross sections and oscillation strengths of magnesium double donor in silicon}

Yu.A. Astrov' ${ }^{1}$, L.M. Portsel' ${ }^{1}$, V.B. Shuman', A.N. Lodygin ${ }^{1}$, N.V. Abrosimov ${ }^{2}$, S.G. Pavlov ${ }^{3}$, H.-W. Hübers ${ }^{3,4}$

${ }^{1}$ loffe Institute, 194021 St. Petersburg, Russia

${ }^{2}$ Leibniz-Institut für Kristallzüchtung (IKZ),

Berlin, Germany

${ }^{3}$ Institute of Optical Sensor Systems, German Aerospace Center (DLR), Berlin, Germany

${ }^{4}$ Institut für Physik,

Humboldt-Universität zu Berlin,

Berlin, Germany

Abstract The optical properties of magnesium impurity in silicon, whose atoms at interstitial positions in the lattice are deep double donors with an ionization energy of $107.56 \mathrm{meV}$ in the neutral state, were studied. For optical transitions from the ground state of a neutral center to the excited levels $2 p_{0}$ and $2 p_{ \pm}$, the absorption cross sections and oscillator strengths were determined. These parameters were calculated from the impurity absorption spectra that were measured at $T \approx 5 \mathrm{~K}$ in samples with different magnesium concentrations. The deep donor content in the samples was determined using Hall effect measurements in the temperature range $78-300 \mathrm{~K}$. The obtained characteristics of intracenter transitions in magnesium were compared with the corresponding literature data for shallow group V donors in silicon, which are substitutional impurities. It was found that the optical characteristics of the investigated transitions in magnesium are consistent with the dependences of the corresponding parameters on the ionization energy for shallow donors, extrapolated to the region of larger electron binding energies. 